\title{
Sunyaev Zel'dovich high resolution view of filamentary structures between galaxy clusters pairs
}

\author{
Federico Radiconi $^{1,2, *}$ for the ACT collaboration \\ ${ }^{1}$ Sapienza University of Rome, Physics Department, Piazzale Aldo Moro 5, 00185 Rome, Italy \\ ${ }^{2}$ INFN - Sezione di Roma, Piazzale Aldo Moro 5 - I-00185, Rome, Italy
}

\begin{abstract}
Strong observational and theoretical evidences suggest that about half of the baryons in the Universe should lie outside galaxy clusters in a lowdensity and hot phase in filaments connecting galaxy clusters. Due to the low density, most of this filamentary plasma can not be detected by X-ray observatories. In particular cases of low redshift cluster pairs in the pre-merging phase, the Sunyaev Zel'dovich (SZ) effect can be used to observe the intercluster regions and detect the imprint of missing baryons.

The Abell 399-401 (A399-401) system is the perfect laboratory to test our ability to detect filamentary structures via the SZ effect with $<\sim 1^{\prime}$ angular resolution. This pair has been well studied at several frequencies: it exhibits double radio-halos, an excess of X-ray emission in the intercluster region and a synchrotron radio 'ridge' connecting the two clusters. Moreover the Planck satellite provided the first SZ detection of the gas between A399-401 despite the poor angular resolution $\left(\sim 10^{\prime}\right)$ of its SZ map.

We have used an Atacama Cosmology Telescope (ACT) and Planck satellite Compton-y map (1.65' angular resolution) that combines ACT data from 2008 to 2019 with Planck maps to study the A399-401 system in detail. We present the data analysis and results.
\end{abstract}

\section{Introduction}

The observed baryon matter fraction in the local Universe is one of the open problems in Cosmology today. Observations of the baryonic matter based on HI absorption, X-ray emission, gas, stars, and galaxies counts provide a smaller baryonic fraction with respect to nucleosynthesis predictions, high-redshift measures of the Lyman-alpha forest absorption, and CMB power spectrum fits $[10,21]$. Hydrodynamical simulations suggest that the fraction of the baryonic matter not directly observable, about half of the total budget, should lie outside galaxy clusters in the form of filamentary structures connecting the clusters forming the socalled cosmic web $[7,23]$. These baryons must be characterized by a low-density phase and temperature in the range: $10^{5}<T<10^{7} \mathrm{~K}$.

The direct observation of missing baryons with X-rays and radio instruments is very challenging because:

- X-ray luminosity scales as the square of the electron density;

\footnotetext{
*e-mail: federico.radiconi@roma1.infn.it
} 
- Outside of galaxy clusters, simulations predict few relativistic electrons and weak magnetic fields, thus making synchrotron emission very faint [24].

The Sunyaev-Zel'dovich (SZ) effect is another method for detecting these baryons; it is due to cosmic microwave background $(\mathrm{CMB})$ radiation inverse Compton scattering off of electrons in a warm or hot gas. The SZ effect is mainly due to two separate components, the thermal and the kinematic one. The thermal component depends only on the gas pressure and it causes a distortion of the CMB spectrum. The kinematic effect is due to the overall peculiar motion of the gas with respect to the CMB. See Mroczkowski et al. (2019) [15] for a recent review about the SZ effect and its applications in astrophysics.

The amplitude of thermal SZ effect at any given frequency is proportional to the Compton- $y$ parameter:

$$
y=\frac{\sigma_{\mathrm{T}}}{m_{\mathrm{e}} c^{2}} \int P_{\mathrm{e}}(r) d r=\frac{\sigma_{\mathrm{T}}}{m_{\mathrm{e}} c^{2}} \int n_{\mathrm{e}}(r) k_{\mathrm{B}} T_{\mathrm{e}}(r) d r
$$

where $\sigma_{\mathrm{T}}$ is the Thomson cross section, $c$ is the speed of light, $m_{\mathrm{e}}$ is the electron mass and $P_{\mathrm{e}}(r)$ is the electron pressure along the line of sight. The thermal SZ effect is proportional to the electron density so SZ measurements of the missing baryons should be easier compared to X-rays and radio observations.

The galaxy cluster pair Abell 399 ( $\mathrm{z}=0.071806$, [17]) and Abell 401 ( $\mathrm{z}=0.073664$, [17]) is the perfect laboratory to investigate the missing baryon problem. The angular separation between the clusters is $36.9^{\prime}$. Assuming a flat $\Lambda$ CDM Universe with $H_{0}=67.6 \mathrm{~km} / \mathrm{s}, \Omega_{m}=$ 0.31 and $\Omega_{\Lambda}=0.69$ [1] and the mean redshift $z=0.072735$, the separation on the plane of the sky between the two clusters is $3.2 \mathrm{Mpc}$. Due to the low redshift, it is possible to disentangle the two clusters at several wavelengths using wide images with $\sim$ arcmin angular resolution. Moreover, in the last decade several studies, at several wavelengths, have reported evidence of the presence of baryons between the two clusters (e.g. [9, 12, 19]). Recently Akamatsu et al. (2017) [3], using Suzaku data, confirmed the existence of hot gas (T $\sim 6-7 \mathrm{keV})$ between A399 and A401. Govoni et al. (2019) [12], using Low Frequency Array (LOFAR) $140 \mathrm{MHz}$ data, observed an excess of radio emission, a 'ridge', between the two clusters. Moreover, they were able to set an upper limit for the magnetic field in the intercluster region, $B<1 \mu G$. The Planck collaboration (2013) [19], using the MILCA Compton-y map with an angular resolution of 7.18', detected an excess of Compton- $y$ signal in the intercluster region despite the poor angular resolution. Bonjean et al. (2018) [4], using new Planck's $y$-map, confirmed previous results and estimated a density between the two clusters of $n_{e}=(4.3 \pm 0.7) \times 10^{-4}$ $\mathrm{cm}^{-3}$.

In these proceedings, we summarise results that appeared in Hincks et al. (2021) [13] focusing on the model fitting and best fits comparison described in that work.

\section{Data}

In this work, we used a Compton- $y$ map from the Atacama Cosmology Telescope combined with existing Planck data. ACT is a 6-metre off-axis Gregorian telescope that started observations in 2008. It is located at 5190 metres in altitude on the Chajnator plateau in the Atacama desert to minimize atmospheric contributions. ACT currently can observe the sky at several frequencies: $30,40,98,150$, and $220 \mathrm{GHz}$ and it can reach an angular resolution between 1 and 2 arcmins at 98, 150 and $220 \mathrm{GHz}$. Together with the South Pole Telescope, ACT is the only high-resolution CMB survey telescope.

In this analysis we used the 98, 150, and $220 \mathrm{GHz}$ data, from 2008 up to 2019, and Planck 100-545 GHz data to construct an ACT+Planck Compton-y map, as explained in Madhavacheril et al. (2020) [14]. The map is showed in Fig. 1 and its main difference with 
Figure 1. Left: ACT Compton- $y$ map of A399-401. The seven external black boxes enclose the regions we used to estimate the covariance matrix. The region surrounded by the red box has been excluded due to dust contamination. Right: Central region of the ACT map. It is the region we fitted with the MCMC in Sec. 3. Contour levels are 3, 5, and $7 \sigma$. The effective beam of the map is showed on the bottom left side as a black circle with a radius equal to half beamwidth.
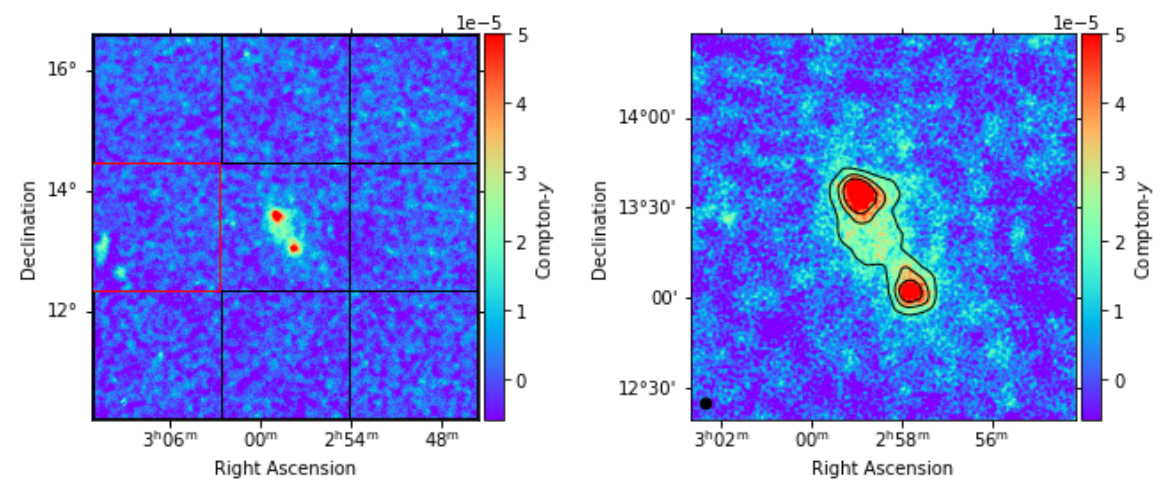

the ACT Data Release 5 (Naess et al. 2020, [16]) is the inclusion of the data acquired during 2019. The map is characterized by an angular resolution of $1.65^{\prime}$ and a noise of $\sigma=5.9 \times 10^{-6} \mathrm{y} /$ pixel, where the pixel size is $0.5^{\prime}$. For further details about the procedure we followed to extract the Compton- $y$ map, see Hincks et al. (2021) [13].

\section{Fitted Models}

In this section, we focus on the fitting procedure we used to model the two galaxy clusters and the intercluster region. We described the two clusters with the isothermal $\beta$-profile [6]:

$$
P(r)=k_{\mathrm{B}} T_{\mathrm{e}} \frac{n_{0}}{\left[1+\left(\frac{r}{r_{\mathrm{c}}}\right)^{2}\right]^{\frac{3}{2} \beta}},
$$

where $k_{B} T_{e}$ is the temperature of the system, $n_{0}$ is the central electron density, $r$ is the distance from the centre of the system, $r_{c}$ is the core radius and $\beta$ parameterizes the slope of the profile. The previous equation describes a spherical system; asphericity can be introduced replacing in Eq. 2:

$$
\frac{r}{r_{\mathrm{c}}} \longrightarrow \frac{\sqrt{x^{2}+(y / R)^{2}}}{r_{\mathrm{c}}},
$$

where $x$ is the coordinate of the major axis, $y$ that of the minor axis and $R$ is the ratio between minor and major axis. To fit the intercluster region we adopted the following model to which we will refer as mesa-model:

$$
g(l, w)=\frac{A_{f i l}}{1+\left(\frac{l}{l_{0}}\right)^{8}+\left(\frac{w}{w_{0}}\right)^{8}},
$$

where $l$ and $w$ represent the axes of a reference frame centred in the center of the mesa, $A_{f i l}$ is the amplitude of the mesa, $l_{0}$ and $w_{0}$ are the typical sizes of the mesa along the two axes. The mesa-model is an ad-hoc model without any physical meaning that has been used to simulate the apparent flatness of the Compton- $y$ signal between the two clusters and sharp edges beyond the $3 \sigma$ contour level, as it is possible to see in Fig. 1. We fitted four different models; each one includes also a plane to remove any residual offsets and gradients: 
- 2 elliptical $\beta$-profiles for the two clusters;

- 3 elliptical $\beta$-profiles for the two clusters and the intercluster region. We fixed the $\beta$ parameter for the intercluster region to $4 / 3$, the value that describes an isothermal cylinder in hydrostatic equilibrium [18];

- 2 circular $\beta$-profiles for the two clusters and the mesa-model for the intercluster region;

- 2 elliptical $\beta$-profiles for the two clusters and the mesa-model for the intercluster region.

\section{Results}

We performed fits of the models described in Sec. 3 using the Niagara supercomputer at the SciNet ${ }^{1}$ HPC Consortium (University of Toronto). We minimized the likelihood (Eq. 6) using emcee [8], a python implementation of the affine-invariant Monte Carlo Markov Chain algorithm [11] using the map region shown in the right panel of Fig. 1. The likelihood of a fitted model is:

$$
\mathcal{L}=\frac{1}{2 \pi|M|^{\frac{1}{2}}} \exp \left(-\frac{1}{2} \mathbf{m}^{T} M^{-1} \mathbf{m}\right),
$$

where $\mathbf{m}$ is the map of residuals and $M$ is the covariance matrix of the map.

We assumed that the fit converges when two conditions are satisfied [8] :

- the length of the chain is greater than $10 \tau$, where $\tau$ is the maximum autocorrelation length for all the parameters;

- the autocorrelation length for each parameter does not increase more than $1 \%$ every 100 iterations.

Once each fit reached convergence, we burned the first $10 \tau$ iterations and we extracted our best fit results using only the remaining set of data. All the best-fit parameters are reported in Table 2 in Hincks et al., (2021) [13]. In Fig. 2 we show the posterior distribution for the '2 Ellip. $\beta+m e s a$ ' in two different subsets to make the figure clearer.

We used two different statistical tests to compare the models: the likelihood ratio and the Akaike Information Criterion (AIC) [2]. The likelihood ratio $W$ is defined as:

$$
W=2 \log \frac{\max \mathcal{L}_{2}}{\max \mathcal{L}_{1}}
$$

where $\max \mathcal{L}$ is the likelihood estimate using best-fit parameters. The likelihood ratio compares two models only if model 2 adds additional parameters to model 1 . In our case, the "base" model is the ' 2 Ellip. $\beta$ ' that is extended by the ' 3 Ellip. $\beta$ ' and the ' 2 Ellip. $\beta+m e s a$ '. The preferred model is the one that maximizes the $W$ parameter. The AIC test is defined as:

$$
A I C=2 K-2 \log (\max \mathcal{L}),
$$

where $K$ are the degree of freedom of the model. According to the Akaike Information Criterion, the preferred model is the one that minimizes the AIC value, which we will refer as $A I C_{0}$. If we define $\Delta_{i}=A I C_{i}-A I C_{0}$, Burnham \& Anderson (2004) [5] suggest that, if $\Delta_{i} \lesssim 2$ the two models have "substantial support", if $\Delta_{i} \gtrsim 4$ the model $i$ has "considerably less support", while if $\Delta_{i} \gtrsim 10$ the model $i$ has "essentially no support". In Tab. 1 we report the results of the statistical tests we did. The likelihood ratio test shows that a bridge component is required to properly fit the data at a level greater than $5 \sigma$ for the ' 2 Elliptical $\beta$-profiles + mes $a$ ' model. The same model is also preferred by the Akaike Information Criterion, having the

\footnotetext{
${ }^{1}$ https://www.scinethpc.ca/
} 
Figure 2. Bottom Left: Posterior distributions for 13 of the 22 parameters that describe the '2 Elliptical $\beta$-profiles $+m e s a$ ' model. Top Right: Posterior distributions for the remaining parameters. Contours levels describe the Gaussian confidence levels at 1,2, and $3 \sigma$.

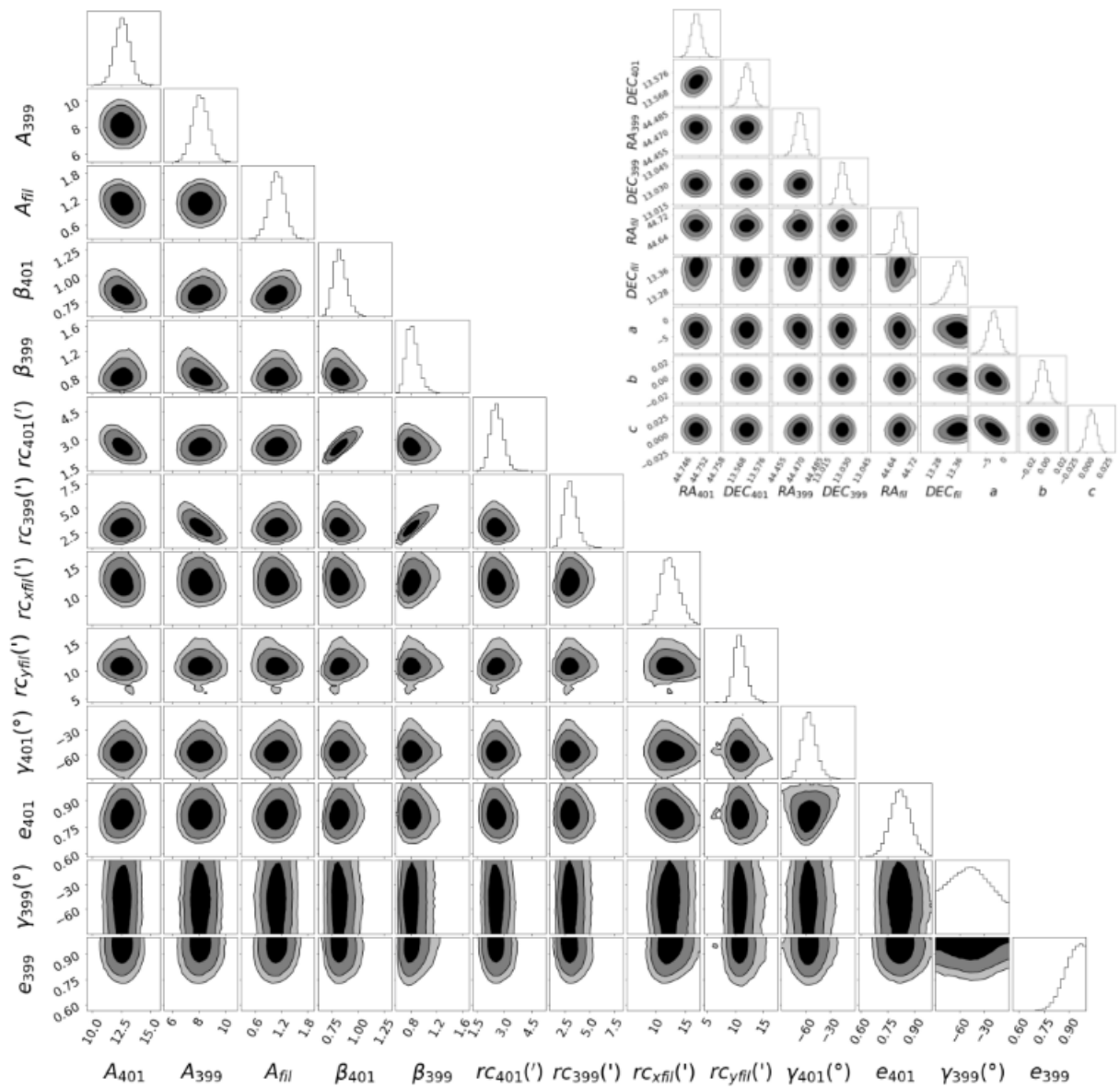

Table 1. The statistical information we used to compare the fitted models.

\begin{tabular}{c|cccccc}
\hline Data & Model & $P$ & \multicolumn{2}{c}{ Likelihood Ratio } & \multicolumn{2}{c}{ AIC } \\
& & & $W$ & $\sigma$ & AIC & $\Delta_{i}$ \\
\hline ACT+Planck & 2 Ellip. $\beta$ & 17 & - & - & 48523.2 & 33.4 \\
& 3 Ellip. $\beta$ & 22 & 33.2 & 4.6 & 48500.0 & 10.2 \\
& 2 Circ. $\beta+$ mesa & 18 & - & - & 48492.2 & 2.4 \\
& 2 Ellip. $\beta+$ mesa & 22 & 43.4 & 5.5 & 48489.8 & 0 \\
\hline Planck only & 2 Ellip. $\beta$ & 17 & - & - & 2976.6 & 13.5 \\
& 2 Ellip. $\beta+$ mesa & 22 & 23.5 & 3.6 & 2961.1 & 0 \\
\hline
\end{tabular}

lowest $\mathrm{AIC}\left(A I C_{0}\right)$. To estimate how higher angular resolution data can improve the detection of the intercluster signal, we fitted the Planck data [20] (PR2 MILCA, 10' angular resolution) with the same ' 2 Elliptical $\beta$-profiles' and ' 2 Elliptical $\beta$-profiles $+m e s a$ ' models.

We estimated the Compton- $y$ signal at the centre of the mesa from our fiducial 2 Ellip. $\beta+$ mesa model, $y_{t o t}=(2.8 \pm 0.3) \times 10^{-5}$, which includes the mesa itself and the clusters' halos. Assuming the temperature measured in the intercluster region by Akamatsu et al. 2017 [3] using Suzaku X-rays observations, $k T_{\text {fil }}=6.5 \pm 0.5 \mathrm{keV}$, they derive the relation 
that links the Compton- $y$ signal with the distance integrated along the line of sight $\left(r_{f i l}\right)$, $y_{\text {tot }}=(8.0 \pm 1.0) \times\left(r_{f i l} / M p c\right)^{0.5} \times 10^{-6}$ (Note that we have corrected an algebraic error from their paper: see Appendix B of Hincks et al. [13]). With the previous $y_{\text {tot }}$ we obtain a thickness along the line of sight of $r_{f i l}=(12.1 \pm 3.9) \mathrm{Mpc}$ and an electron density of $n_{e}=(0.88 \pm 0.24) \times 10^{-4} \mathrm{~cm}^{-3}$. About half of this density comes from the cluster halos, while the remaining part is due to the filament, implying the density in the intercluster region to be $\sim 150$ times the mean baryon density of the Universe at $z=0.07$. Moreover, the typical sizes of the mesa on the plane of the sky are $l_{0} \times w_{0}=(2.2 \times 1.9) \mathrm{Mpc}$, much smaller than the thickness along the line of sight, indicating that the system does not lies entirely on the plane of the sky as it was previously assumed [3]. For a more detailed study about the geometry of the system see Hincks et al. (2021) [13].

\section{Conclusions}

We have shown that using ACT+Planck maps, with an effective angular resolution of $1.65^{\prime}$, we improved the significance level of the detection of a Compton- $y$ signal between A399 and A401 with respect to Planck only data. Our best fit model consists of 2 Elliptical $\beta$-profiles for A399 and A401 and a mesa function (Eq. 4) between the two clusters. From our fiducial model combined with X-ray data we have evidence that the system lies mostly along the line of sight. We will further investigate this system with new ACT data, not included in this work.

\section{References}

[1] Aiola S., et al., J. Cosmology Astropart. Phys., 047 (2020)

[2] Akaike H., IEEE Trans. Automat. Contr., 19, 716 (1974)

[3] Akamatsu H., et al., A\&A, 606, A1 (2017)

[4] Bonjean V., Aghanim N., Salomé P., Douspis M., Beelen A.,A\&A, 609, A49 (2018)

[5] Burnham K. P., Anderson D. R., Sociol. Methods Res., 33, 261 (2004)

[6] Cavaliere A., Fusco-Femiano R., A\&A, 70, 677 (1978)

[7] Cen R., Ostriker J. P., AJ, 514, 1 (1999)

[8] Foreman-Mackey D., Hogg D. W., Lang D., Goodman J., PASP, 125, 306 (2013)

[9] Fujita Y., Koyama K., Tsuru T., Matsumoto H., PASJ, 48, 191 (1996)

[10] Fukugita M., Hogan C. J., Peebles P. J. E., AJ, 503, 518 (1998)

[11] Goodman J., Weare J., CAMCoS, 5, 65 (2010)

[12] Govoni F., et al., Science, 364, 981 (2019)

[13] Hincks A., et al., arXiv:2107.04611 (2021)

[14] Madhavacheril M. S., et al., Phys. Rev. D, 102, 023534 (2020)

[15] Mroczkowski T., et al., Space Sci. Rev., 215, 17 (2019)

[16] Naess S., et al., J. Cosmology Astropart. Phys., 2020, 046 (2020)

[17] Oegerle W. R., Hill J. M., 2001, AJ, 122, 2858 (2001)

[18] Ostriker J., ApJ, 140, 1056 (1964)

[19] Planck Collaboration, $A \& A$, 550, A134 (2013)

[20] Planck Collaboration, $A \& A, 594$, A22 (2016)

[21] Planck collaboration, $A \& A, 641, \mathrm{~A} 6$ (2020)

[22] Sunyaev R. A., Zel'dovich Y. B., CoASP, 4, 173 (1972)

[23] Tuominen T., et al., A\&A, 646, A156 (2021)

[24] Vazza F., Ferrari C., et al $A \& A$, 580, A119 (2015) 